Article

\title{
Improving the Energy Efficiency of Petrochemical Plant Operations: A Measurement and Verification Case Study Using a Balanced Wave Optimizer
}

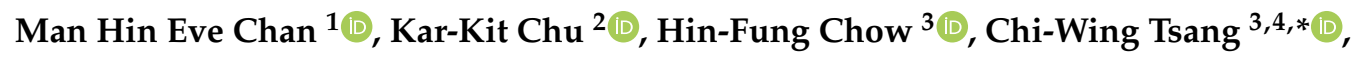 \\ Chi Kuen Danny Ho ${ }^{5}$ and Shuk-Kei Ho ${ }^{3}$ (D) \\ 1 Faculty of Design and Environment, Technological and Higher Education Institute of Hong Kong (THEi), \\ Hong Kong 999077, China; evechan@vtc.edu.hk \\ 2 Campus Management and Development Office, University of Macau, Taipa, Macau 999078, China; \\ garychu@um.edu.mo \\ 3 Faculty of Science and Technology, Technological and Higher Education Institute of Hong Kong (THEi), \\ Hong Kong 999077, China; 160614089@stu.vtc.edu.hk (H.-F.C.); gaye.ho@hotmail.com (S.-K.H.) \\ 4 Research Centre for Waste and Resource Management, Hong Kong 999077, China \\ 5 Department of Supply Chain and Information Management, The Hang Seng University of Hong Kong, \\ Hong Kong 999077, China; dannyho@hsu.edu.hk \\ * Correspondence: ctsang@vtc.edu.hk
}

Received: 10 September 2019; Accepted: 25 October 2019; Published: 30 October 2019

\begin{abstract}
The Chinese petrochemical industry is facing pressure to meet strict targets of energy consumption and carbon emission reductions. Water pumps are the primary equipment used in most chemical and agrochemical industries sectors since water is commonly used for cooling and heating purposes, but these pumps also consume a large amount of energy. Other uses of water pumps in these industries include producing steam for heating, preparing reaction media or absorptive reagents, rinsing products, and distilling. As for the electrical components of the water pump systems, current technologies of variable frequency drives and superconducting transmission lines are unable to increase the energy efficiency of these systems with a fixed load. However, the Balanced Wave Technology (BWT) is offered as a solution to overcome these limitations. In this report, a case study using a BWT optimizer is conducted on a closed loop water circulation system. Two BWTs are added to the individual motor-controlled section of each pump that is being used on the switchboard. For the first time, a detailed example was provided on how to implement option B of the International Performance Measurement and Verification Protocol (IPMVP) in China by evaluating the performance of BWT as an energy conservation measure. The evaluated periods included those of the baseline, post-installation, and actual performance of the optimizers. An average saving of energy of about $10.46 \%$ is recorded in a 5 -week reporting period. On this basis, that annual electricity saved is estimated to be $66,447.18 \mathrm{kWh}$, which is equivalent to the emission of 68.94 metric tons of $\mathrm{CO}_{2} \mathrm{e}$. This case study demonstrates in detail how option B of IPMVP can be implemented for BWTs applied on pumping systems. In addition to petrochemical production plants, other industries like textile and clothing sections, which are heavy users of water and electrical energy with fixed loads in the production processes of raw materials, fiber, yarn, and fabric, as well as textile-dyeing and final treatment, could benefit from applying this new technology.
\end{abstract}

Keywords: Electrical Energy Reduction; Motor Driven Pump; Compton Effect; Industrial Energy Audit; IPMVP Option B 


\section{Introduction}

Modern society is very reliant on the different petroleum-derived products for daily life products, as reflected in global chemical sales, which approximated $€ 3.4$ trillion in 2016 and are expected to reach $€ 6.3$ trillion in 2030 [1]. As a rapidly growing economic and industrial powerhouse, China is the largest global producer of various chemicals and chemical-related commodities. Its share of chemical production accounted for nearly $40 \%$ worldwide in 2016 and is predicted to contribute to half of the currently expanding market by 2025 [1,2]. Undoubtedly, the petrochemical industry, as one of the most important industry pillars of the economy of China, will continue to grow. However, the production of petrochemicals is an intensive process that involves extreme temperatures and pressures, thus resulting in energy-intensive and capital-intensive manufacturing processes. As the largest energy user, this sector comprised $28 \%$ of the worldwide industrial final energy consumption in 2014, and the process of energy use accounted for $42 \%$ [3]. Hence China is determined to focus on green development in this industry to meet its target announced at the 2015 Paris Climate Change Conference, which is a reduction of $60 \%-65 \%$ in carbon intensity based on its 2005 levels by 2030 [4]. The State Council of the People's Republic of China established the key targets on 3 August 2016 for an $8 \%$ reduction in energy consumption and $10 \%$ reduction in carbon dioxide $\left(\mathrm{CO}_{2}\right)$ per $¥ 10,000$ of industrial added value based on the levels for the 12th Five-Year Plan (2011-2015) [5]. In addition, China intends to invest more than $1.2 \%$ of the taxation revenues from this industry in innovative research and development to support energy efficiency [5]. The findings of a report by the International Energy Agency indicated that an increase in energy efficiency between 2000 and 2016 reduced global energy use by $12 \%$ [6]. Comparatively speaking, global electricity demand in 2017, which increased by $3.1 \%$, is significantly higher than the $2.1 \%$ increase in global energy demand [7]. For such an energy-intensive industry, the challenge of further carbon emission reductions cannot solely rely on the use of renewable alternatives to replace fossil feedstocks. Consequently, increasing electrical energy efficiency in the major energy consumption sources by introducing energy-saving technologies could be the solution to help reduce the target gap.

Improving power factors in industrial set-ups is one way to improve energy efficiency [8], which in turns improve the long-term economic and environmental gains to the society [9]. However, the high cost of investment in capacitor banks is always an obstacle to their wide-spread use among the industries [10]. A practical measure to increase energy efficiency is to minimize power loss in current electric motor-driven systems, such as water pumps. Pumps comprise more than $20 \%$ of the total electricity consumption in China [11]. The petrochemical industry has the highest rate of use of pumps compared to other industries, which constitutes around 51\% of total number of pumps in use [12]. In petrochemical plants, water pumps are often integrated with heat exchangers to increase heat transfer efficiency. The electricity used by the water pump systems is the fluid power divided by the product of all components efficiencies due to the energy loss incurred from the electrical input power, motors, power transmission, and pumps. The energy loss from the motor pumps is assumed to be about $40 \%$ without considering losses from the transmission lines [13]. Although the value seems to be quite high, the reasons for the pump inefficiencies vary due to various pump subcomponent inefficiencies. These can be due to the working fluid's viscosity, vapor pressure and density, operating temperature and pressure, net positive suction head, motor controller [14], electricity transmission, etc. Most current practices for improving the efficiency of pumping systems on the electricity consumption aspects focus on reduction of large energy losses from the mismatch between the output power of fixed speed motors and the demanded mechanical power of the electromechanical systems. In situations where fixed speed pumps operate in place but are not fully loaded, the use of variable frequency drives (VFDs) could reduce energy consumption from $30 \%-50 \%[12,15,16]$. VFDs can ramp up and down the frequency and voltage to adjust the speed so that the load of the electric motor can be met. The generation of power that is not used and is therefore waste can be avoided. Moreover, the operation of this speed-controlled system would require a considerable amount of energy itself. If the flow rate cannot be varied nor reduced, this application will, in turn, incur more energy loss and 
impose higher costs to maintain a constant output system [12,17]. Other methods such as impeller trimming, replacing with pumps with smaller loads, or switching off extra pumps [18] do not offer a holistic approach to reducing the overall energy consumption. To address this particular problem, optimization of the system with alternative technology for electrical input power could be realized by using Balanced Wave Technology (BWT) which could provide noticeable energy reductions to systems with both variable and fixed loads It is expected that BWT will be particularly applicable to systems with fixed loads, such as the water pump system used in petrochemical plants, since it may take a shorter induction period and reach stable conditions as soon as possible. Therefore, a water pump system for providing process water to a catalytic reactor is chosen as a demonstration in this case study. Besides applicability, the more serious problem is the substantial energy losses incurred by the power distribution network that provides electricity from the substations to the loads. The energy losses from power lines have been underestimated at around 3\%, which is the same as those from fuel pipes, but the distribution losses from fuel pipes and electricity lines have been reported to be as high as $10 \%-40 \%$ in the manufacturing and mining sectors in the United States [13]. The electricity transmission losses are primarily caused by the electrical resistance of the conductor lines, in which the resistance comes from the friction of electrons that bump into the atoms, which prevents electron transfer capability. The cables that carry a higher current, sustain more heat through resistive losses because of the Joule effect which thereby causes higher energy loss. Even though the correct cable size can minimize the loss, some inherently technical losses in the form of heat and noise occur when the system is energized. Thus, BWT could provide an energy saving options as a whole, rather than to each particular parts of the pump system, such as the motors, motor couplers, pump, valves, pipes, and the control system [19]. Current case study reports are very limited. We are aware of a few industrial retrofit examples with variable and fixed loads, however, few details have been revealed including the details of auditing methodology, all parameter measurement [option B of International Performance Measurement and Verification Protocol (IPMVP), and the system stability after switching off the optimizer. Indeed, in order to consider BWT as one potentially popular energy conservation measures (ECM) in future, more details need to be included.

Up to now, detailed examples on the use and performance reporting of BWT to improve energy efficiency have remained very scarce, in particular the details of the auditing procedure and stability during and after the test based on measurement and verification plan are rarely reported. The utilization of economic feasible BWT to reduce resistive losses in electricity lines would be invaluable to the petrochemical industry in China so that it would remain competitive in the global marketplace. This case study demonstrated an example of using BWT to reduce the energy consumption of a water pump system at a petrochemical production plant in China without significantly modifying the existing hardware or affecting the product output. On the other hand, China still faces substantial challenges in the area of energy saving measurement and verification. Although China has issued national standards/protocols for energy saving calculation and guidelines for the most commonly used energy efficiency technologies, these standards and protocols are not sufficiently detailed to provide operational guidance to the 3rd party verifiers [20]. While the system of measurement and verification in China is still under development $[20,21]$, for the first time, we provided an example on how to implement option B of the International Performance Measurement and Verification Protocol (IPMVP) in China by evaluating the performance of BWT as the energy conservation measures. The full details on the auditing methodology, parameter measurement, and the system stability after switching off the optimizer are reported in this paper.

In the following parts of this report, the principle of BWT will first be briefly mentioned. Previous case studies will then be introduced and the flaws of these reported studies will be briefly mentioned, which are the data insufficiency and the performance instability. After the methodology is introduced, the data acquisition process is described followed by data presentations. Finally, a concluding remark and the future prospects of this application are discussed. 


\section{Methods}

\subsection{Balanced Wave Technology}

In electricity transmission, energy is lost due to heat, vibration, noise, and electromagnetic radiation over electric cables. Balanced Wave Technology (BWT) is a patent technology registered in 2008 by KSE Co. Ltd. for saving power in electric circuits by maximizing the strength of electric currents [22]. The larger the conductivity of the current, the smaller the resistant exits. The strengthened currents can improve energy efficiency because of minimizing power losses incurred in resistance materials. This is achieved by a diminution of de-orbited electrons to reduce the number of electromagnetic waves, as well as a diminution of friction between moving electrons to reduce the frictional vibration, heat, and noise [23]. The basic concepts behind this technology comprise the law of the photoelectric effect, the law of Compton effect, the law of photochemical effect, and the characteristics of optical ceramic, specifically lead lanthanum zirconate titante (PLZT) together [23]. The researched material of PLZT is light-absorbing ferroelectric ceramics that have a potency to generate a high density of power and energy [24]. Through the application of PLZT, a substantial light source can be accumulated in Balanced Wave Technology. In light-matter interaction, an alternation of light intensity would induce a change of kinetic energy in the electrons radiated from the metal. Photons can alter the momentum and movement of free electrons to center the flow along the conductor and elongate the wavelength of free electrons, thereby lengthening the electric current. The working principle of BWTs is by infrared ray synthetic wavelengths irritated on a semiconductor, which is formed with PLZT and optical ceramic, then emitted a pulse micro photocurrent to a transmission line connected from a power source to a load [22]. The testing of BWTs in this study integrated a current overload protection system. In addition, the safety certifications for BWTS were acquired from Underwriters Laboratories Inc. (UL), Conformity of Europe (CE) and TÜV Rheinland Group (TÜVs).

Some case studies of the application of BWTs in electric motor driven water pump systems are shown in Table 1. The reduction in energy use ranged from $8.6 \%$ to $12.0 \%$ [25-27]. The BWTs worked best on a continuously operating motor for a certain period. The kick-in period (induction period) varied from 8 weeks to 16 weeks depending on the motor efficiency, the old and new conditions of the system and equipment, the operational routine, and the cleanliness of the grid. The energy efficiency of the system was gradually improved due to the accumulative effect of the BWTs' effort. Thus, of the three company systems retrofitted with the BWTs, the operational length and continuity of SNC-Lavalin obtained the most energy savings. Although the Hebei Datang International Zhangjiakou Thermal Power Co. Ltd. had employed the BWTs for 12 weeks, with good electric power quality, relatively new equipment and the system being in good condition, it still took a longer optimal time than that of the general system [25]. Other than saving energy, the BWTs effectively reduced the heat of motors by approximately $2{ }^{\circ} \mathrm{C}$, thereby extending the life of motors [25]. The temperatures of the transmission system were lower when less transmission power dissipated in the form of heat energy. The line resistance increased linearly with increasing temperatures [28]. So, the line resistance was reduced as the line temperatures have decreased. This proved the BWTs reduced the friction of electrons. However, in all these three cases, other key parameters to determine the performance of the retrofit such as water pressure, temperature and flow rates are not reported. 
Table 1. Case studies on energy improvements by Balanced Wave Technologies (BWTs).

\begin{tabular}{llll}
\hline Company & $\begin{array}{l}\text { Hebei Datang International } \\
\text { Zhangjiakou Thermal } \\
\text { Power Co. Ltd. }\end{array}$ & Siam Centre & SNC-Lavalin \\
\hline Function & $\begin{array}{l}\text { Industry } \\
\text { A closed loop water } \\
\text { circulation system with } 2\end{array}$ & Shopping Complex & Office \\
Retrofit System & water pumps in parallel & & $\mathrm{kW}$ chilled water pumps \\
System Features & $2 \times 185 \mathrm{~kW}$, fixed load & $3-4$ pumps run in rotation & $25 \mathrm{Hp}$ water chiller pump motor \\
BWT Model & $2 \times 200 \mathrm{kVA}$ & $135 \mathrm{kVA}$ & $600 \mathrm{~V}, 1770 \mathrm{rpm}, 60 \mathrm{~Hz}$, fixed load \\
Operating Hours/Day & - & 12.5 hours & $25 \mathrm{kVA}$ \\
Operating Days/Year & 7 days & 7 days & 24 hours \\
Performance Period & 12 weeks & 8 weeks & 7 days \\
Baseline kWh Used & $302.70 \mathrm{kWh}$ & $5446 \mathrm{kWh}$ per 5 days & $22.21 \mathrm{kWh}$ per hour \\
Post Retrofit $\mathrm{kWh}$ & $276.76 \mathrm{kWh}$ & $4864 \mathrm{kWh}$ per 5 days & $19.55 \mathrm{kWh}$ per hour \\
Energy Savings $(\%)$ & $8.57 \%$ & $10.69 \%$ & $12.0 \%$ \\
\hline
\end{tabular}

\subsection{The Auditing Procedure}

In order to perform the test with more reliable results, a comprehensive field test of Balanced Wave Technology was conducted for a period of 19 weeks on a closed loop water circulation system at a petrochemical plant in China, in which the monitoring is longer than the previous case study (Siam Centre). The reason for this is that although this example had comparable energy savings and \% performance, whether the pump system was run with fixed or variable loads was not known. It is expected that with variable loads, the optimizer will take a much longer induction period. The test was run for only 8 weeks and it was thus unclear how long the induction period is and whether the performance can be sustained after a longer period of time. The testing requirements and the calculation of energy saving rates followed by the standard of the People's Republic of China "SY/T 6422-2008-The measuring for energy conservation effect of energy conservation products in petroleum enterprise". This specific standard applied to a unified technical requirement of the oil and gas industry for quantifying the energy efficiency of inventions in the given conditions. To fulfil the standard and to evaluate the actual value of the BWTs in this application, the test was carried out in normal day-to-day operation at the plant without modifying the system and production rates. In this case, the plant throughput maintained a continually fixed load to create a substantial output. As such, the water pump system was running non-stop 24/7/365 in a constant load for a year barring a necessary yearly cycle of an overhaul. The pumps stopped in a complete overhaul interval of about 42 days just after 10 weeks of BWTs had started. The BWTs required an initial period of working continuously for 8-12 weeks to achieve an expected energy reduction of a minimum of $10 \%$. To compensate for the suspension during testing, the $10 \mathrm{~mm}^{2}$ wires were replaced by $16 \mathrm{~mm}^{2}$ wires to improve the conductivity before re-starting the installation. This promoted a greater current-carrying capacity between the BWTs and the loads. The testing system was a parallel arrangement of three $50 \%$ centrifugal pumps for pumping a high volume of cooling water to the heat exchangers. In the parallel mode, the inflowing water entered the eyes of both impellers together at the same pressure and was discharged from both impellers together into a common delivery. The BWTs was directly connected in parallel to the distribution box closest to the water pumps. The benefit of the parallel design was to ensure the safe operation of the target equipment to the utmost extent. Two feedwater pumps P1601/1 and P1601/2 of model CZS100-250 were driven by electric motors of output power $55 \mathrm{~kW}$ apiece with an efficiency of $93 \%$ at $380 \mathrm{~V}, 50 \mathrm{~Hz}$. The third pump P1601/3 of model PC150-3315-25 was used as a backup. The transmission distance of electricity from an indoor main distribution switchboard to the water pumps was less than 50 meters.

At the switchboard, a BWT was added by a $40 \mathrm{~A} 3$ poles miniature circuit breaker and $10 \mathrm{~mm}^{2}$ wires to an individual motor control section of each in-use pump for electricity enhancement. The length between both connections was 5 meters. The installation of BWTs was parallel to the incoming load and outgoing load [29]. This protected the water pumps system from being interrupted when BWT 
failed. Even if the BWTs failed, the device would immediately be isolated by the added circuit breaker, so normal production would not be affected.

\subsection{Acquisition of Data}

The data comparison before and after energy saving based on the entire water circulation system. These included the energy consumption of the two pumps (denoted as P1601/1 and P1601/2 in Figure 1), the two flow meters measuring the water flow rates (denoted as FIC417 and FI701 in Figure 1), the pressure meter (denoted as PI411 in Figure 1), the two thermocouples measuring the temperatures of return and influent water (denoted as TI426 and i TI427 in Figure 1). A calibrated meter of three-phase power analyzer "Circutor CVM-NRG96" was installed in an individual power distribution cabinet of each in use pump to measure the main electrical parameters. The magnitudes of active power $(\mathrm{kW})$, reactive power (kvar), apparent power $(\mathrm{kVA})$, power factor, load factor $(\%)$, current $(\mathrm{A})$, voltage $(\mathrm{V})$, current unbalance rate (\%), and voltage unbalance rate (\%) were generated by the meters at 10 second intervals for 24 hours continuously. The recording data were stored at the connected computer. On the same day, the factory installed two power meters of model "DTSD341-Three Phase Static Multi-Function Smart Energy Meter" at the nearly same location for accurate verification. All computerized data of pumps P1601/1 and P1601/2 were taken using a daily and hourly mean separately and were used in the calculation consistently throughout an assessment period. Water flow rates $(\mathrm{t} / \mathrm{h})$, pipeline pressure $(\mathrm{MPa})$, and temperatures $\left({ }^{\circ} \mathrm{C}\right)$ of inflowing and returned water produced by factory's meters in $1 \mathrm{~min}$ to $3 \mathrm{~min}$ were also used for analysis in energy use. The calculation of energy saving rates was based on a unit energy consumption in a unit flow rate under $1 \mathrm{MPa}$.

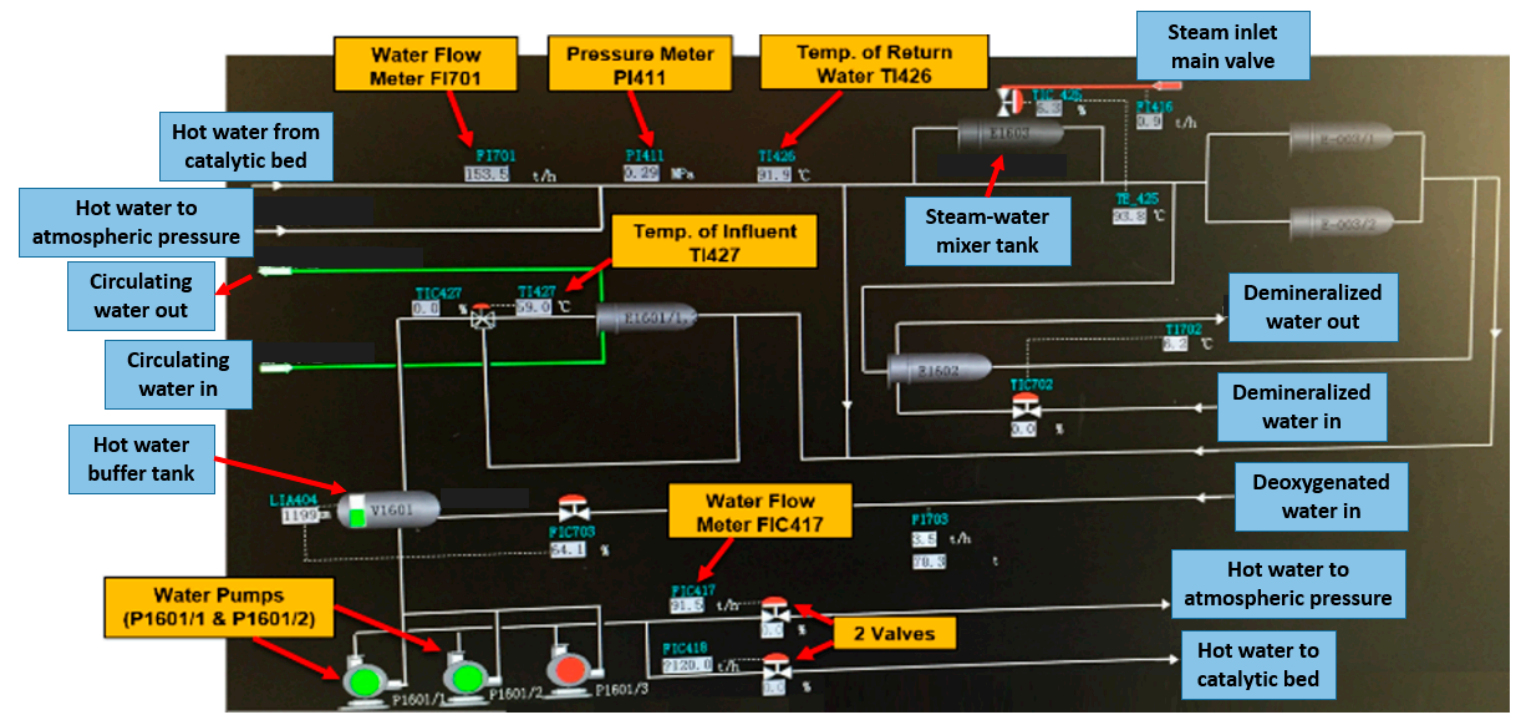

Figure 1. The water circulation system.

The three main testing periods for assessment were: a baseline development, the introduction phase, and actual performance of the BWTs. The baseline period was audited for 7 days before the BWTs were installed. The baseline data were utilized to evaluate the effectiveness of the BWTs in every stage and to choose the BWTs with suitable load capacity. During the baseline, load factors of the pumps P1601/1 and P1601/2 were at least 98.9\%. The equation of load factor (LF) per day is expressed in Equation (1).

$$
\mathrm{LF}(\%)=\frac{\text { Average Active Power }(\mathrm{kWh})}{\text { Maximum Active Power }(\mathrm{kWh})} \times 100 \%,
$$

The high LF indicated energy use in the water pump system was relatively constant. The maximum apparent power of P1601/1 and P1601/2 were $52.13 \mathrm{kVA}$ and $51.22 \mathrm{kVA}$ for reference. Then, the two 
BWTs with a load capacity of $60 \mathrm{kVA}$ were installed. Supplying currents in the electrical circuit passed to the BWT and then to the installed meter before going to the motor, as shown in Figure 2. After installation, the induction period began and was tested for 10 weeks until the overhaul interval. The actual work of the BWTs was not achieved. Once the maintenance of factory completed, the two BWTs were restarted and continued to run for another 8 weeks, except for a temporary shutdown of the BWT in P1601/1 because of some production issues. The first two to three weeks after the restart were an adjustment of water flow rate until it began to stabilize. The BWTs sometimes required an adjustment returning to the pre-stop status after the restart from being shut down for a long time. Therefore, the reporting period of its actual performance was 5 weeks.

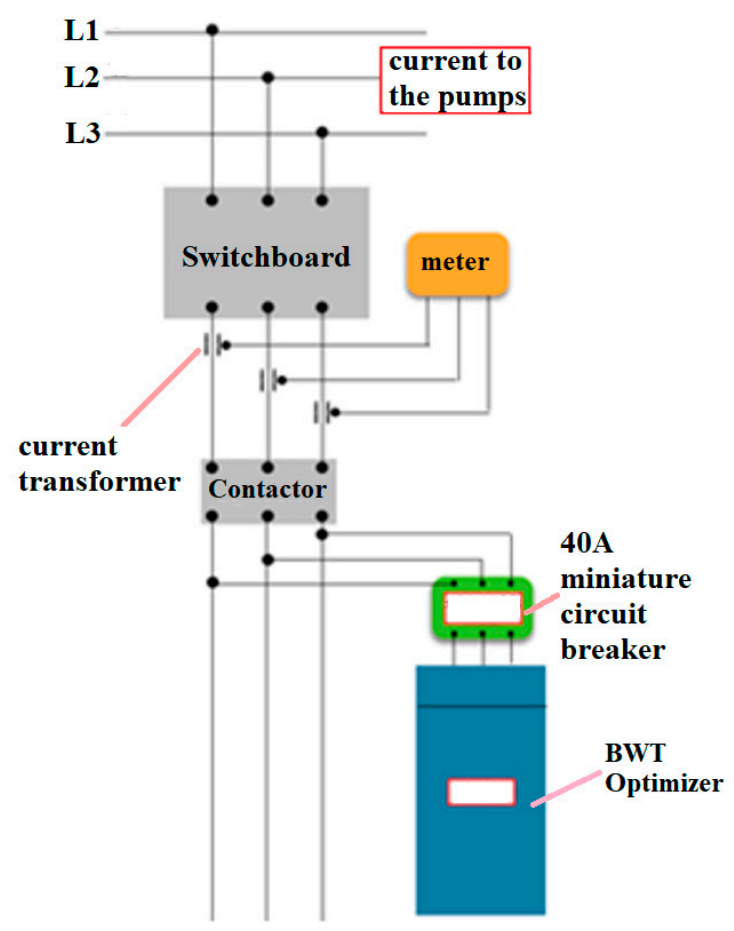

Figure 2. A wiring connection of BWT and meter.

\subsection{Data Manipulation}

The comparative results were established for the stable operation of the water pumps. All adopted data were within $5 \%$ disparity between the highest and lowest water flow rates. The deviation of energy data sets generated by the two contrasting models of meters was $\pm 0.1 \%$ and is expressed as Equation (2).

$$
\text { Difference }(\%)=\frac{\text { First set value }- \text { Second set value }}{(\text { First set value }+ \text { Second set value }) / 2} \times 100 \%
$$

The daily average unit of energy consumption per hour is calculated by Equations (3) and (4). It measured the power supply for each tonne of fluid flow in the water pipes. The measurement of active power was to evaluate the amount of power that the pumps required to perform the work. The measurement of reactive power was to evaluate the amount of power transferred within the alternative circuit. By using both power components, the ultimate avoided energy can be computed by Equation (5) [30]. The given equation was abstracted from the standard of SY/T 6422-2008 for specifically counting the energy saving rate after the pumps retrofit.

$$
\text { Average Active Power }\left(\mathrm{kWh} / \mathrm{m}^{3}\right)=\frac{\text { Average } 1-\mathrm{hr} \mathrm{kW}}{[\text { Average flow rate }(\mathrm{t} / \mathrm{h}) \div \text { Average } 1-\mathrm{hr} \text { Pressure }(\mathrm{MPa})]} \times 100 \% \text {, }
$$


Average Reactive Power $\left(\mathrm{kvarh} / \mathrm{m}^{3}\right)=\frac{\text { Average } 1-\mathrm{hr} \text { kvar }}{[\text { Average flow rate }(\mathrm{t} / \mathrm{h}) \div \text { Average } 1-\mathrm{hr} \text { Pressure }(\mathrm{MPa})]} \times 100 \%$,

$$
\xi_{b}=\left[\frac{w_{1}-w_{2}+\mathrm{K}_{q}\left(Q_{1}-Q_{2}\right)}{w_{1}+\mathrm{K}_{q} Q_{1}}\right] \times 100 \%
$$

where $\xi_{\mathrm{b}}=$ comprehensive energy-saving rate, $\%$;

$\mathrm{W}_{1}=$ active power before BWTs have started, $\mathrm{kWh} / \mathrm{m}^{3}$;

$\mathrm{W}_{2}=$ active power after BWTs started, $\mathrm{kWh} / \mathrm{m}^{3}$;

$\mathrm{Q}_{1}=$ reactive power before BWTs have started, $\mathrm{kvarh} / \mathrm{m}^{3}$;

$\mathrm{Q}_{2}=$ reactive power after BWTs started, $\mathrm{kvarh} / \mathrm{m}^{3}$;

$\mathrm{K}_{\mathrm{q}}=$ reactive economic equivalent, $0.03 \mathrm{~kW} / \mathrm{kvar}$ for the requirement of GB/T 12947-2006.

The logging intervals of water flow rates and pipeline pressure were 1 to $3 \mathrm{~min}$, which differed from logging intervals of energy parameters $(10 \mathrm{~s})$. The data sets with 1 - to 3-minute intervals were less accurate than those with $10 \mathrm{~s}$ intervals, while the margin of error for a sample proportion in the final energy saving was $\pm 1 \%$ and is defined as Equation (6).

$$
\text { Margin of error }(\%)=\left[Z \times \sqrt{\frac{\hat{p}(1-\hat{p})}{n}}\right] \times 100 \%
$$

where $\hat{\mathrm{p}}=$ sample proportion; $\mathrm{n}=$ sample size; $\mathrm{z}=\mathrm{z}$-score of $99 \%$ confidence.

For electricity cost and carbon footprint reduction, the following formula were adopted:

$$
\begin{gathered}
5 \text { - week } \mathrm{kWh}-\text { saving rate }(\%)=\left[\frac{w_{1}-\left(\frac{\sum w_{2}}{5}\right)}{w_{1}}\right] \times 100 \% \\
\mathrm{CO}_{2} \text { electricity }=\mathrm{EF}_{e} \times \text { Activity }_{e^{\prime}}
\end{gathered}
$$

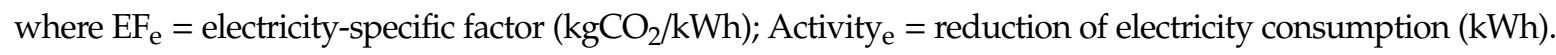

\subsection{Option of Measurement and Verification Method}

The goal of the water pump system retrofit was to reduce the motor's energy use by at least $10 \%$ on average without altering the operational criteria of the existing structure. The measurement and verification $(\mathrm{M} \& \mathrm{~V})$ method of energy reduction was option B of International Performance Measurement and Verification Protocol (IPMVP) [31]. A helping guideline of IPMVP was first developed under the U.S. Department of Energy in 1994 to report verifiable savings for water and energy uses with four measurement options [32]. By using option B, all of the energy consumption and operational parameters were continuously metered directly in the factory to minimize uncertainty [28]. These parameters comprised short-term data logging of motor energy consumption, pipeline pressure and water flow rate to verify and quantify energy efficiency improvements. The adopted power logger apparatus measured amperage input and voltage input then calculated power factor, active energy, reactive energy and apparent energy. This power logging method gave a highly accurate energy consumption data with either short or long intervals [33]. The water temperatures were also monitored to verify that the system was maintained at the desired set points. These measurements were taken during the baseline, post-installation of the BWTs, and its actual performance. Because of the water pumps run with a constant speed at full-load operation in a year. The chosen comparable baseline was one-week period. The performance of the BWTs was the difference of energy inputs between the baseline and post-retrofit. Thus, the assessment of energy savings from the BWTs was simply the baseline energy minus the reporting-period energy with no adjustment needed. 


\section{Results and Discussion}

\subsection{Baseline Energy Consumption}

The average hourly of 7 days baseline energy usage of P1601/1 and P1601/2 was $0.1162 \mathrm{kWh} / \mathrm{m}^{3}$ and $0.0681 \mathrm{kvarh} / \mathrm{m}^{3}$. The average power factor of the pumps was 0.8618 . A summary of evaluation data during the baseline period is shown in Appendix A.

\subsection{Initial Energy Savings}

The 10 weeks of BWTs have started but the actual performance was not yet reached. The energy saving in this period was being monitored. The calculation of the comprehensive saving rate was omitted because of 7 days of power data that was lost between week 7 and week 8 . The avoided energy in the first 8 weeks was less than $1 \%$. The reactive power had a faster dropping rate than the active power from week to week. On week 10, a large reduction in active power led to $5.7 \%$ energy savings. A brief outcome is shown in Table 2. The average power factor in this period was 0.9537.

Table 2. Initial comprehensive energy-saving rate.

\begin{tabular}{|c|c|c|c|c|c|}
\hline Week & $\mathrm{W}_{1}\left(\mathrm{kWh} / \mathrm{m}^{3}\right)$ & $\mathrm{W}_{2}\left(\mathrm{kWh} / \mathrm{m}^{3}\right)$ & $\mathrm{Q}_{1}\left(\mathrm{kvarh} / \mathrm{m}^{3}\right)$ & $\mathrm{Q}_{2}\left(\mathrm{kvarh} / \mathrm{m}^{3}\right)$ & $\xi_{b}(\%)$ \\
\hline 1 & \multirow{10}{*}{0.1162} & 0.1161 & \multirow{10}{*}{0.0681} & 0.0372 & 0.87 \\
\hline 2 & & 0.1171 & & 0.0373 & 0.04 \\
\hline 3 & & 0.1166 & & 0.0367 & 0.48 \\
\hline 4 & & 0.1164 & & 0.0363 & 0.64 \\
\hline 5 & & 0.1166 & & 0.0361 & 0.55 \\
\hline 6 & & 0.1165 & & 0.0375 & 0.55 \\
\hline 7 & & 0.1162 & & 0.0365 & 0.85 \\
\hline 8 & & 0.1162 & & 0.0347 & 0.88 \\
\hline 9 & & 0.1159 & & 0.0343 & 1.14 \\
\hline 10 & & 0.1106 & & 0.0323 & 5.70 \\
\hline
\end{tabular}

\subsection{Overall Energy Savings}

After the extensive overhaul, the whole system required regular calibration. Individual variations within 8 weeks after the overhaul in FIC417, FI701, P1411, TI426, and TI427 were significant, as shown in Figure 3. Nevertheless, the relative standard deviations in total flow rate, pressure and total temperature in the adopted 5 -week period were $1.7 \%, 1.1 \%$, and $1.2 \%$ accordingly. The differences in total flow rate and pressure were included in the calculation of the unit energy consumption.

Each week of the 5-week reporting period had over 10\% of avoided energy as shown in Table 3. The average comprehensive energy saving rate was $10.46 \%$. The energy saving rate fluctuated slightly every week resulting from the regular shutdown of BWT for P1601/1 per factory request. This resulted in an average power factor of 0.9279 lower than for the initial stage. The reactive power usage increased gradually once the function of BWT was turned off. In contrast, the active power usage increased only in week 5 . The unilateral increase in reactive power caused the power factor to decrease. The interruption of the test cycle and the insufficient running time of BWT might have slightly affected the energy-saving effect and this effect could have been underestimated. 


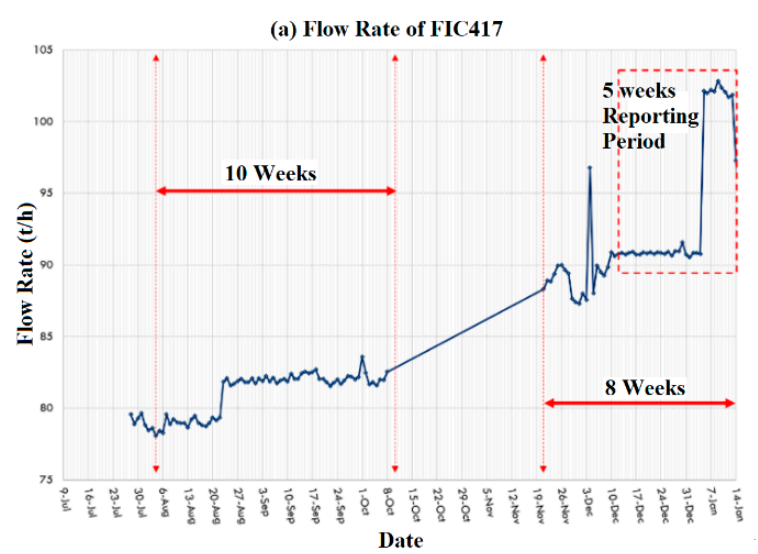

(c) Pressure of PI411

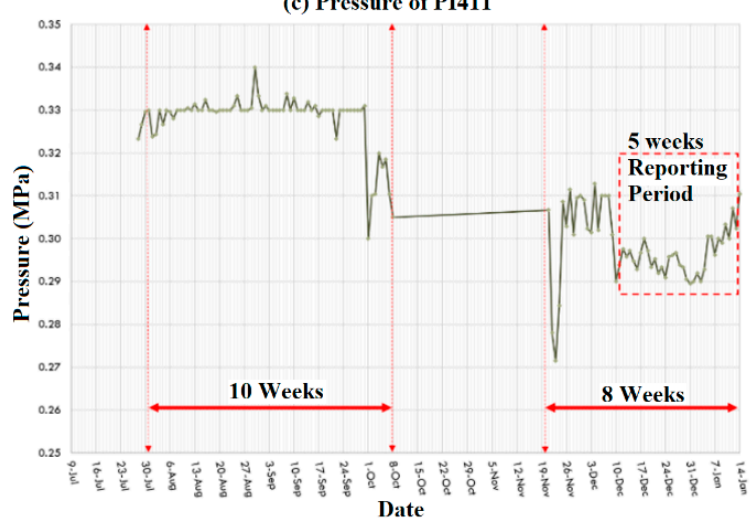

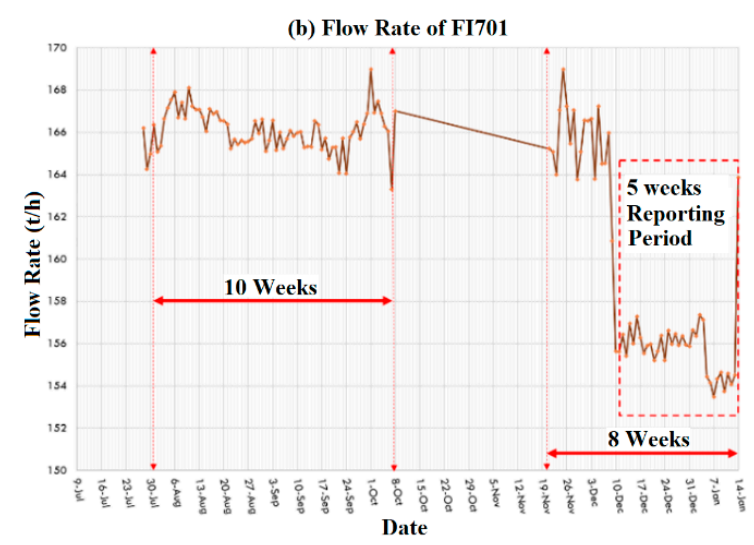

(d) Temperature of Influent and Return Water

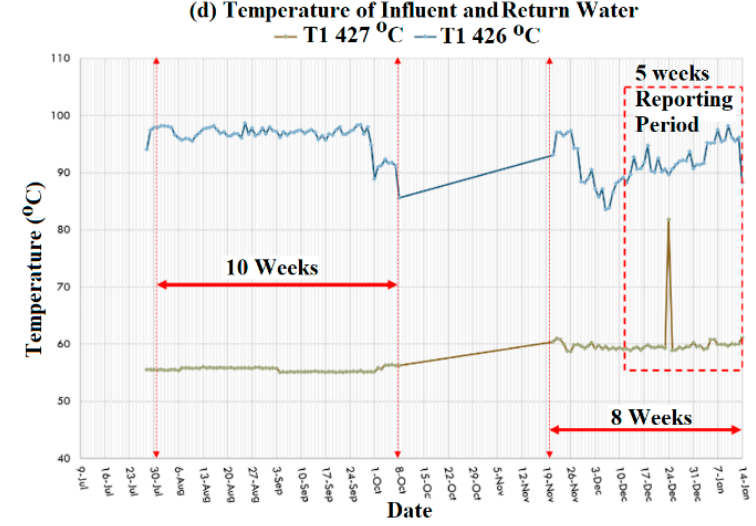

Figure 3. An overview of the system performance in the testing period: (a) flowrate of FIC417; (b) flowrate of FI701; (c) pressure of PI411; (d) temperature of influent and return water.

Table 3. Final comprehensive energy-saving rate.

\begin{tabular}{lccccc}
\hline Report Week & $\mathbf{W}_{\mathbf{1}}\left(\mathbf{k W h} / \mathbf{m}^{3}\right)$ & $\mathbf{W}_{\mathbf{2}} \mathbf{( \mathbf { k W h } / \mathbf { m } ^ { 3 } )}$ & $\mathbf{Q}_{\mathbf{1}}\left(\mathbf{k v a r h} / \mathbf{m}^{3}\right)$ & $\mathbf{Q}_{\mathbf{2}}\left(\mathbf{k v a r h} / \mathbf{m}^{3}\right)$ & $\boldsymbol{\xi}_{\mathbf{b}}(\mathbf{\%})$ \\
\hline 1 & & 0.1053 & & 0.0320 & 10.15 \\
2 & & 0.1053 & & 0.0365 & 10.08 \\
3 & 0.1162 & 0.1043 & 0.0681 & 0.0446 & 10.66 \\
4 & & 0.1038 & & 0.0452 & 11.10 \\
5 & & 0.1047 & & 0.0457 & 10.34 \\
\hline
\end{tabular}

The diagrams of energy parameters for P1601/1 and P1601/2 in Figures 4-6 showed the changes in energy consumption after activation of BWTs. Active power consumption was directly proportional to the total water flow rate, as shown in Figure 4. Active power of both P1601/1 and P1601/2 tended to level out in the first 10 weeks, and the usage declined gradually from the beginning of the reporting period until a sudden rebound with the increase of total flow rate. The fall of active power was not as obvious as that of reactive power, but the usage decreased noticeably before the rise.

Instead, the reactive power dropped significantly and sharply as shown in Figure 5, and the power factor reached above 0.95 when the BWTs remained on, as shown in Figure 6. The rise of power factor drew less current at the same time. That was because the reactive power was being diminished. The reactor power continuously bounced back and forth between the source and load causing heat energy within the wire. The reduction of reactive power and improving power factor illustrated that the power was being consumed more effectively. Once the BWT switched off on P1601/1, the power factor slumped immediately, and the reactive power returned to its original level. This was in stark contrast to P1601/2, where reactive power had been maintained constantly. These findings indicated that the BWTs had a gradual influence on active power over a period and a consistently immediate effect on reactive power. 

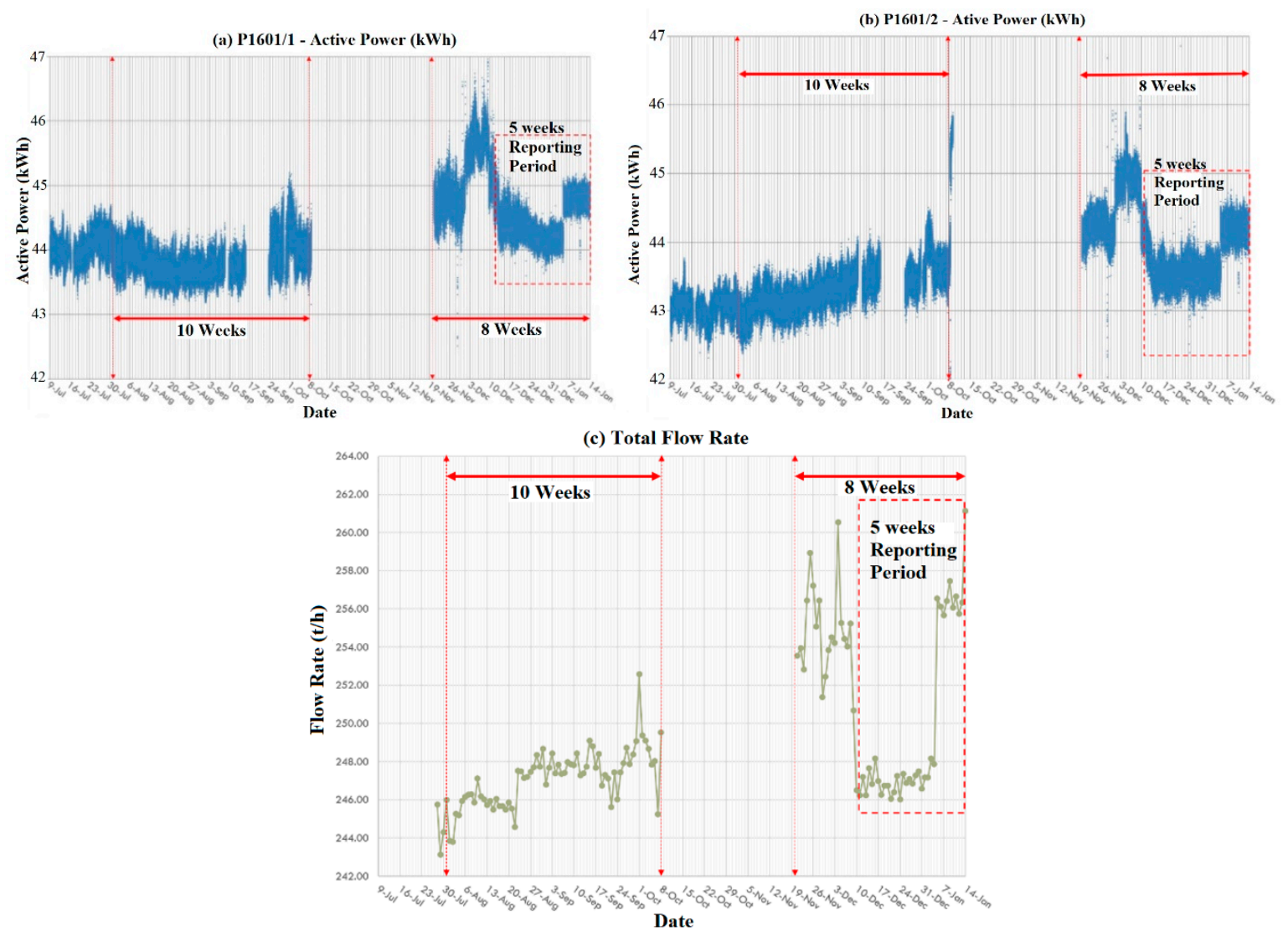

Figure 4. Active power of (a) P1601/1 and (b) P1601/2 related to (c) total water flow rate.
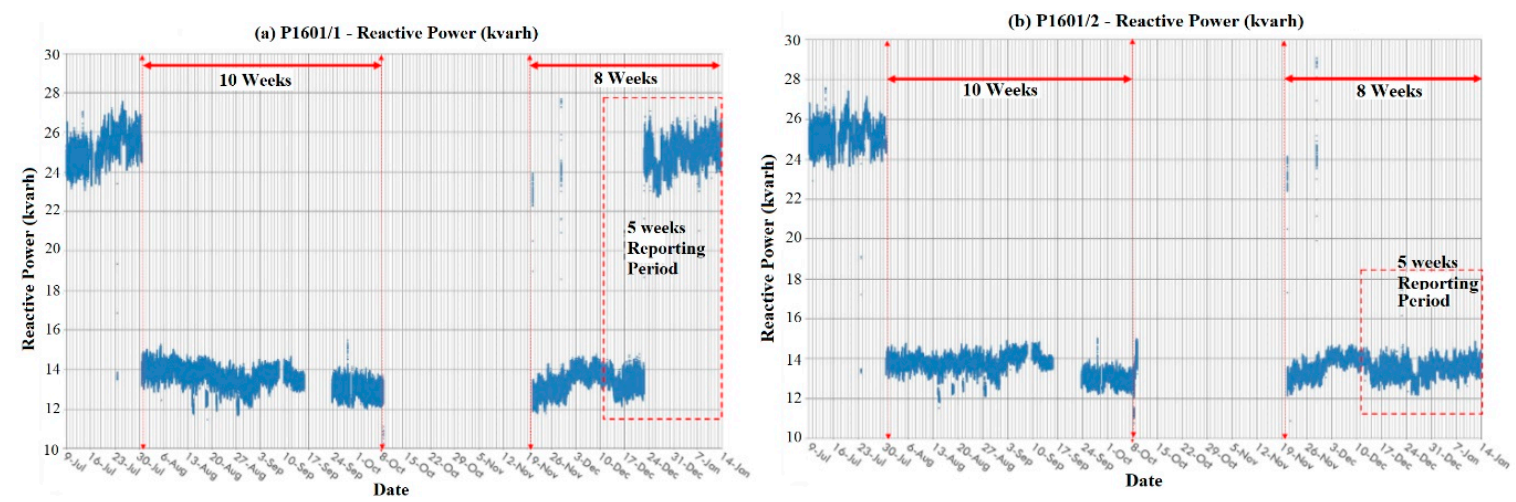

Figure 5. Reactive power of (a) P1601/1 and (b) P1601/2.

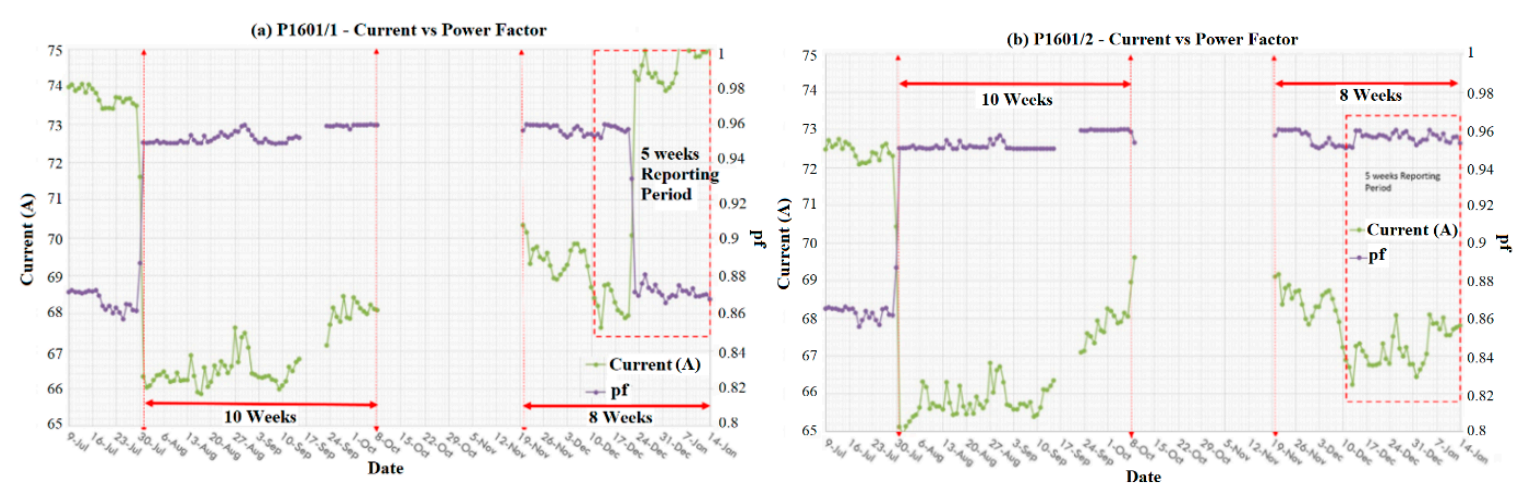

Figure 6. Current compared with a power factor of (a) P1601/1 and (b) P1601/2. 
By using BWTs the electricity consumption was lowered, leading to a decrease in carbon emission. The average baseline electricity use by the pumps was $87 \mathrm{kWh}$. The annual running time was $24 \mathrm{~h}$ a day, multiply a year minus 45 days' overhaul. The 5-week avoided active power was $9.94 \%$ and the $\mathrm{kWh}$ saving was calculated using Equation (7) so the total electricity savings in a year was $66,447.18 \mathrm{kWh}$.

For a typical average industrial electricity cost of $¥ 0.6561 / \mathrm{kWh}$ in China, the annual potential saving on this project was $¥ 43,595.99$ [34]. In additon, $68,938.08 \mathrm{~kg}$ of $\mathrm{CO}_{2}$ emissions could be reduced due to lower electricity usage. The emissions were estimated by Equation (8), with an electricity-specific emission factor of 1.03748694, which accounted for the $\mathrm{CO}_{2}$ emissions per $\mathrm{kWh}$ of electricity consumed in China [35].

\section{Conclusions}

Our experimental results demonstrated the energy saving potential of BWTs on the continuously operated fixed speed water pumps. For the first time, we have demonstrated the use of option B of the IPMVP to perform a comprehensive study on energy saving using BWT technology, and we monitored all the parameters. The results were evaluated by reliable data from direct measurement and monitoring, and the procedure was sufficiently detailed to provide operational guidance to the $3 \mathrm{rd}$ party verifiers during the project implementation stage. Since pumps are one of the major sources of energy consumption in petrochemical plants, improving pump efficiency can yield significant energy savings, cost savings, and carbon emissions avoidance. Significantly, Balanced Wave Technology was verified to be an energy-saving approach that was not affected by the water pressure, water flow, and other working conditions of the petrochemical plant. An average saving of energy of about $10.46 \%$ is recorded in a 5-week reporting period. On that basis, that annual electricity saved is estimated to be $66,447.18 \mathrm{kWh}$, which is equivalent to the emission of 68.94 metric tons of $\mathrm{CO}_{2} \mathrm{e}$. In particular, for pump P1601/2, reactive power had been maintained constantly, even it was switched off.

The technology improved the quality of currents and the conductivity of electrical circuits to increase motors' output power without changing operational conditions. The BWTs reduced the reactive power generated by inductive loads prominently and hence increased the power factor. According to the working principle and working characteristics of the BWTs, the BWTs should have good energy saving effects for most equipment in the petrochemical plant, such as air compressors, fans, and other common equipment driven by electric motors. Therefore, the next step will obviously be to extend the study to other areas in the petrochemical plant to increase the overall energy savings of the plants and to identify which operation units work best with BWT. BWTs in the petrochemical field should be a new green energy saving approach that can help the industry to tap the potential of energy saving in a new way.

This study demonstrates the economic and environmental benefits of BWTs with a case study of its application in a petrochemical plant. It can be envisioned that the full potential of BWTs can be attained in manufacturing processes that heavily rely on extensive water pump systems with high electricity consumption. In addition to petrochemicals, other industrial sectors like textiles and clothing, which is a heavy user of water and electrical energy in the production of raw materials, fiber, yarn, and fabric as well as textile-dyeing and treatment processes, could benefit from applying this new technology. Without the need for changing existing hardware and output and significant investments, BWTs present a quick and handy way to pursue sustainable production through reduced energy consumption. This kind of energy-saving technology is particularly relevant for developing countries like China, which lag behind in the development of renewable energy sources, to attain carbon reduction target in the short run without slowing down economic development.

Author Contributions: Conceptualization, K.-K.C. and C.-W.T.; Funding acquisition, C.-W.T.; Methodology, M.H.E.C. and K.-K.C.; Supervision, K.-K.C. and C.-W.T.; Validation, H.-F.C.; Visualization, C.K.D.H.; Writing-original draft, S.-K.H.; Review \& editing, C.-W.T. 
Funding: This work was fully supported by Research Grants Council (RGC) of the Hong Kong Special Administrative Region, China (Project No.: UGC/IDS25/15) and the Research Centre of Waste and Resource Management.

Acknowledgments: The authors acknowledge the Research Centre of Waste and Resource Management at Technological and Higher Education Institute of Hong Kong (THEi) for administrative and technical support.

Conflicts of Interest: The authors declare no conflict of interest.

\section{Appendix A}

Table A1. Table of Baseline data.

\begin{tabular}{|c|c|c|c|c|c|c|c|c|c|}
\hline \multirow[t]{2}{*}{ Day } & \multicolumn{2}{|c|}{$\begin{array}{c}\text { Active Power } \\
\text { (kWh) }\end{array}$} & \multicolumn{2}{|c|}{$\begin{array}{c}\text { Reactive Power } \\
\text { (kvarh) }\end{array}$} & \multicolumn{2}{|c|}{ Flow Rate (t/h) } & \multirow{2}{*}{$\begin{array}{c}\begin{array}{c}\text { Pressure } \\
\text { (MPa) }\end{array} \\
\text { PI411 }\end{array}$} & \multicolumn{2}{|c|}{ Temperature $\left({ }^{\circ} \mathrm{C}\right)$} \\
\hline & P1601/1 & P1601/2 & P1601/1 & P1601/2 & FI701 & TI427 & & TI427 & TI426 \\
\hline 1 & 43.84 & 43.02 & 24.74 & 25.23 & 166.1810 & 78.2952 & 0.3262 & 55.5095 & 97.9048 \\
\hline 2 & 43.89 & 43.04 & 25.42 & 25.95 & 166.1810 & 78.5333 & 0.3300 & 55.5286 & 98.1000 \\
\hline 3 & 43.94 & 42.94 & 25.63 & 25.48 & 166.4238 & 78.5667 & 0.3300 & 55.5095 & 97.7667 \\
\hline 4 & 43.91 & 42.83 & 25.52 & 25.03 & 164.5238 & 78.3333 & 0.3300 & 55.5333 & 98.6143 \\
\hline 5 & 44.00 & 43.96 & 25.86 & 25.33 & 166.5357 & 78.5929 & 0.3200 & 55.3714 & 94.0143 \\
\hline 6 & 44.17 & 43.10 & 25.64 & 25.12 & 166.4095 & 79.8619 & 0.3233 & 55.5000 & 93.6000 \\
\hline 7 & 44.21 & 43.17 & 25.97 & 25.67 & 165.7381 & 79.4381 & 0.3300 & 55.5000 & 96.1667 \\
\hline Average & 43.99 & 43.01 & 25.54 & 25.40 & 165.9990 & 78.8031 & 0.3271 & 55.4932 & 96.5952 \\
\hline Total & \multicolumn{2}{|c|}{87.0} & \multicolumn{2}{|c|}{50.94} & \multicolumn{2}{|c|}{244.8020} & & & \\
\hline
\end{tabular}

\section{References}

1. Hadhri, M. Facts E Figures 2017 of the European Chemical Industry; Cefic's Facts \& Figures Publications: Brussels, Belgium, 2017; pp. 6-7.

2. Graham, L. Chinese Petchem Exports upset World Markets; ChinaOil: Hong Kong, China, 2017; pp. 1-18.

3. International Energy Agency. Tracking Clean Energy Progress 2017; International Energy Agency: Paris, France, 2017; pp. 1-116.

4. NRDC. The Road from Paris: China's Progress toward Its Climate Pledge; NRDC: New York, NY, USA, 2017; pp. 1-5.

5. China Government. China to Improve Petrochemical Industry; China Government: Beijing, China, 2016.

6. International Energy Agency. Energy Efficiency 2017; International Energy Agency: Paris, France, 2017; pp. 1-143.

7. International Energy Agency. Global Energy \& $\mathrm{CO}_{2}$ Status Report 2017; International Energy Agency: Paris, France, 2018; pp. 1-15.

8. Lotsu, S.; Yoshida, Y.; Fukuda, K.; He, B. Effectiveness of a Power Factor Correction Policy in Improving the Energy Efficiency of Large-Scale Electricity Users in Ghana. Energies 2019, 12, 2582. [CrossRef]

9. Costantini, V.; Crespi, F.; Paglialunga, E. The employment impact of private and public actions for energy efficiency: Evidence from European industries. Energy Policy 2018, 119, 250-267. [CrossRef]

10. Adefarati, T.; Oluwole, A.S.; Olusuyi, O.; Sanusi, M.A. Economic and industrial application of power factor improvement. Int. J. Eng. Res. Technol. 2013, 2, 1945-1955.

11. No leakage pump into the mainstream of petrochemical pumps. In Enterprise News; Ningbo Zhenguan Electrical Co. Ltd.: Ningbo, China, 2015.

12. Fleiter, T.; Eichhammer, W. Energy Efficiency in Electric Motor Systems: Technology, Saving Potentials and Policy Options for Developing Countries; United Nations Industrial Development Organization: Vienna, Austria, 2012; pp. 1-49.

13. U.S. Department of Energy. Energy Use, Loss and Opportunities Analysis: U.S. Manufacturing E Mining; U.S. Department of Energy, E3M Incorporated: Washington, DC, USA, 2004; pp. 1-169.

14. United States Department of Energy. Improving Pumping System Performance-A Sourcebook for Industry; United States Department of Energy: Washington, DC, USA, 2006. 
15. Hebri, S. Variable frequency drive importance in an LPG plant: Case study. Electr. Electron. Syst. 2012, 1, 1-4. [CrossRef]

16. Variable Speed Pumping-A Guide to Successful Applications, Executive Summary; Hydraulic Institute, Europump, U.S. Department of Energy: Washington, DC, USA, 2004; pp. 1-22.

17. Murray, M. Total System Efficiency. In Power Transmission Engineering; Randall Publications LLC: Elk Grove Village, IL, USA, 2010; pp. 16-23.

18. Punwassi, V. Energy Savings through Pipe Header Optimization on a Cooling Tower Pumping Station. Energy Eng. 2016, 114, 63-76. [CrossRef]

19. Bredin, M.S. Optimization of Building and Agricultural Pumping System Energy Efficiency for Maximum Return on Investment. Energy Eng. 2017, 114, 11-32. [CrossRef]

20. Johnson, T.M. Developing Market-Based Energy Efficiency Program in China; World Bank: Washington, DC, USA, 2017.

21. Energy Efficiency Measurement and Verification Issues and Options-An Overview of International Measurement and Verification Experience; World Bank: Washington, DC, USA, 2013.

22. Kwon, Y.-D. Electric Power Saving Apparatus Comprising Semi-Conductor Device to Pass Energy of Infrared Ray Synthetic Wavelength into Electric Cable Using Output Pulse Signal, Electric Circuit Board Structure for Implementing the Apparatus, and Electric Power Saving Method. US Patent 7,373,535 B2, 13 May 2008.

23. Cho, S.-H.; Choi, M.-H.; Kwon, Y.-D.; Kim, C.-D. A study on improving electric energy result by using composition wavelength. In Proceedings of the 3rd IEEE International Conference on Industral Informatics (INDIN'05), Perth, WA, Australia, 19 December 2005; pp. 566-571.

24. Lee, F.Y.; Jo, H.R.; Lynch, C.S.; Pilon, L. Pyroelectric energy conversion using PLZT ceramics and the ferroelectric-ergodic relaxor phase transition. Smart Mater. Struct. 2013, 22, 1-16. [CrossRef]

25. Yuan, H.T. Application of Energy Optimizer in Motor Energy Saving, China. 2013. Available online: www.chinaqking.com (accessed on 5 September 2019).

26. Power Optimizer Case Study in Montreal, Canada. Power Optimizer. Available online: http://www. poweroptimizer.com (accessed on 5 September 2019).

27. Power Optimizer Case Study in the Siam Center, Thailand. Power Optimizer. Available online: http: //www.poweroptimizer.com (accessed on 5 September 2019).

28. Anumaka, M.C. Analysis of technical losses in electrical power system (nigerian $330 \mathrm{kv}$ network as a case study). IJRRAS 2012, 12, 320-327.

29. Comparison between E-Clean Energy Saver and Conventional Energy Controller Installation Method; One Energy Holdings Pte Ltd.: Singapore, 2013.

30. National Energy Administration. The measuring for energy conservation effect of energy conservation products in petroleum enterprise. In Oil and Gas Industry Standard of the People's Republic of China; National Development and Reform Commission: Beijing, China, 2008; pp. 1-7.

31. International Performance Measurement and Verification Protocol, Concepts and Options for Determining Energy and Water Savings; U.S. Department of Energy Office of Scientific and Technical Information: Oak Ridge, TN, USA, 2016; Volume 1.

32. Overview of Different Measurement and Verification (MEV) Protocols; Natural Resources Canada's CanmetENERGY: Québec, Canada, March 2018; pp. 1-20.

33. Harding, A.C.; Nutter, D.W. Measurement and verification of industrial equipment: Sampling interval and data logger considerations. Energy Eng. 2016, 113, 7-33. [CrossRef]

34. Pollitt, M.G.; Yang, C.H.; Chen, H. Reforming the Chinese Electricity Supply Sector: Lessons from International Experience; Energy Policy Research Group, University of Cambridge: Cambridge, UK, 2017; pp. 1-70.

35. Brander, M.; Sood, A.; Wylie, C.; Haughton, A.; Lovel, J. Electricity-Specific Emission Factors for Grid Electricity; Ecometrica: Edinburgh, UK, 2011; pp. 1-22.

(C) 2019 by the authors. Licensee MDPI, Basel, Switzerland. This article is an open access article distributed under the terms and conditions of the Creative Commons Attribution (CC BY) license (http://creativecommons.org/licenses/by/4.0/). 